\title{
LANGUAGE PROFICIENCY TESTING AND THE CONTRASTIVE ANALYSIS DILEMMA
}

\author{
J. A. Upshur \\ University of Michigan
}

The idea of using contrastive analyses in writing foreign language tests has provided a working hypothesis for the actual construction of a number of instruments, and has given considerable impetus to a more general concern for sounder language testing. Heurism is not, however, a sufficient criterion for the adequacy of a theory, so, before accepting contrastive analysis as a valid theory of language testing, its assumptions and implications should be explored. Although not related to theoretical validity, the practical utility of the contrastive analysis hypothesis should also be explored. It is the purpose of this paper to map out such an exploration and indicate some of the things which may be found.

As a minimum, three assumptions are crucial to contrastive analysis and language testing-two relating to the learning process and one to the applicability of the analyses to the testing situation.

1. The first assumption is that there is in fact a different process in learning a second language from that of learning one's native language-a difference more fundamental than the obvious differences in learning situations, age of the learner, etc.

2. The second assumption underlying the contrastive analysis hypothesis centers about a conceptualization of this difference between native and second language learning. This difference is primarily one of learning new responses to previously discriminated stimuli. In the process of learning his native language the child learns to make discriminations among the total range of universally common phones in order that he can use his own sound system. In second language learning, he must learn which of all the already discriminable phones are functionally identical; that is, he must learn to give an identical response to all of the allophones of each target language phoneme unconsciously, and must learn to use only target language phones 
appropriately in his speech. The concepts, abstractions and relationships (i.e., the denotata) for which his native language provides lexical or syntactic signs have already been learned. Second language learning at this level is primarily a process of learning a new set of signs and sign processes. In other words, when a learner of a foreign language concentrates upon what he wants to communicate or upon a message being communicated, he makes habitual linguistic responses. Before he has learned the foreign language, these responses are habits of his own language. As he proceeds to learn the foreign language he makes fewer and fewer mistakes; he is able to make habitual target language responses rather than responses determined by the structure of habits in his native language. Thus the learning of target-language responses is influenced to a great degree by transfer of training. In general, transfer may be considered as a tendency to make a habitual response in a novel situation as a function of the similarity between the stimulus of the old habit and the stimulus of the new situation. Both the degree of similarity of the stimuli and of the two responses affect the difficulty of learning new habits. Sometimes an old habit will apply in a new situation or will aid in learning the new habit. This is positive transfer. At other times the old habit is not applicable. This is negative transfer.

3. The third assumption of the contrastive analysis hypothesis is that contrastive analyses can identify the target language habits for which learning will be unnecessary or will be facilitated by positive transfer and those for which learning will be interfered with because of negative transfer. Furthermore, the degree of interference can also be ascertained by these analyses.

Depending upon one's particular aims and upon the theory and data he brings to bear, these three assumptions will admit implications of virtually infinite extension. Considering, however, only that languages are different and accepting a few elementary and generally accepted principles of testing, we may list three implications of the contrastive analysis hypothesis which will illustrate major practical and theoretical problems.

(1) The first implication (following from assumption 2 above) is that different language backgrounds will present different transfer problems in the learning of any foreign language.

(2) When applied to the problem of second language testing, a second implication of these assumptions is that testing the 
language is most effectively and efficiently accomplished by testing the transfer problems identified by the contrastive analyses. The number of problems not mastered would then give a measure of the amount of learning still to be accomplished. This measure is then used to construct a scale of target-language proficiency. This implication will be explored further below.

(3) There is a further implication of this hypothesis in case a test is to be used for groups of students representing more than one language background. Because different language backgrounds present different learning problems, a test would have to contain items testing the problems peculiar to each language group tested. Furthermore, inasmuch as some groups will have more numerous and more tenacious learning problems, the test would have to be constructed to reflect this difference. This means that the test must have problems for each language group proportional to the total number and difficulty of problems that exist for that group.

When more than two or three native language groups are to be tested (groups with different problems as well as common problems with different difficulty) the problem of balancing such a test becomes extremely complex. In fact, it becomes so complex that it requires almost unlimited time or the use of electronic computers. This, however, is a matter of practical utility, not of theoretical validity.

Because the problem of creating a balanced test for many different language backgrounds is so difficult, and because the problem composition of the test depends upon the specific groups to be tested, it might be better to construct one test which would sample proportionally all of the problems for all languages. This is, in fact, what is accomplished when an examination is written from an analysis of the target language alone.

There are two general approaches to language proficiency testing: one emphasizing learning and the other performance. The ideal of the performance approach would be to put the examinee in the target language environment and see how well he can get along-how well he can communicate and comprehend in his role in the environment. Because this is obviously a highly impractical method for assessing language proficiency, tests are constructed to reflect the linguistic situations the subject is likely to encounter. His performance in these artificial situations is assessed, and his proficiency in the wider, natural environment inferred.

The learning approach begins with a determination of the specific responses and skills a person must learn if he is to 
become proficient in a particular foreign language. The test is then constructed to measure control of these analyzed responses and skills.

It should be apparent that these two approaches are not perfectly discrete, and that most tests fall somewhere on a continuum somewhere between the two extremes. Certainly specific responses must be learned if one is to get along in a foreign language environment, and the responses and skills one learns are used in the natural environment. Nonetheless, the test constructor is faced with the problem of interpretation and so attempts to write his test from one or the other of these approaches. The degree to which a particular test falls short of either extreme is usually a compromise dictated by practical considerations of time, cost, availability of adequate analyses, etc. In discussing theory, however, practical limitations may be presumed not to exist.

The contrastive analysis hypothesis applies to learningapproach testing. It is the purpose of the analyses to identify which specific factors must be learned; these learning problems then constitute the domain of the test. As stated in assumption 2 above, the learning problems are transfer problems-instances in which there will be negative transfer from the native to the target language. The first implication of the contrastive analysis hypothesis, implication (1) above, is that different language backgrounds, because of their differing linguistic habit structures, will present different transfer problems in the learning of the target language.

Herein lies a dilemma, a logical inconsistency in the contrastive analysis hypothesis. As the student begins to learn the new language, his linguistic habit structure changes, and it is this altered habit structure which will determine the amount and locus of negative transfer in subsequent learning. After any small increment of learning, the student is no longer the "pure native speaker" assumed by the contrastive analysis of the native and target languages. All of what he has learned will have facilitation or interference effects upon what has not yet been taught. The units of the measuring scale, responses to be learned, have been changed. Therefore, analogous to the situation of testing students of many native language backgrounds, it is necessary to have the test balanced for students with the same native language background but with different orders or amounts of targetlanguage learning.

The dilemma may be more formally stated: A test is a scale with units equally applicable to all subjects being measured by the scale, which is used to order those subjects with 
respect to some property which the scale is designed to measure. The contrastive analysis hypothesis is valid for subjects having the same native language background and the same amount of knowledge of the target language. Subjects having the same native language and learning backgrounds will achieve identical test scores. Therefore, either the test will not order subjects or the contrastive analysis hypothesis is invalid.

It is not necessary to conclude from the foregoing that contrastive analysis is valueless. It is an impractical method for determining test content when students from many language backgrounds are to be tested, and it is a theoretically invalid method for determining test content when students of a single native language background are to be tested; but the theoretical invalidity of the hypothesis does not mean that it is useless in the construction of language tests. It only means that results of tests constructed by this method will be subject to errors of interpretation when viewed as strict measures of comparative learning required of the examinees. It means that a source of error has been built into the test.

It is certainly too fond a hope that any single theory or approach to language testing could apply equally to all testing situations at this early time in the development of second language testing. Accordingly, when developing any testing rationale or constructing any test, account must be made of the kind of test wanted and of the specific testing situation: Is the test to be an aptitude test, a proficiency test, or an achievement test? Is the test to be used for screening, for diagnosis, or for purely pedagogic purposes? Will the subjects to be tested be homogeneous with respect to language background, educational level, age? Is the test to be "informal" or will it be the end result of an extensive program of writing, analysis of experimental tests, and validation? Evaluation of the limitations of any hypothesis will depend in large degree upon the answers to these and other questions. 